\title{
Letter to editor: A Proposal to Mitigate the Consequences of Type 2 Error in Surgical Science
}

We read the article by Babebekov et al. published under Surgical Perspectives with great interest. ${ }^{1}$ There is no doubt that inadequate power to detect significant findings, or type 2 error, is a concern in surgical science, especially when the interpretation of the results are made inadequately. This belongs to fundamental reasons behind the so called "replication crisis"., 3 As the authors write, absence of evidence is not evidence of absence. The authors conclude that CONSORT and STROBE guidelines should "include the disclosure of power with the given sample size and effect size observed in that study".

When stipulating power using the study sample size and observed effect size, one is calculating the post-hoc power. The authors make a serious and well known error in their article when suggesting the use of post-hoc power to mitigate type 2 error. Statistical power (a priori power usually) is the probability of finding a significant effect, if there is a true difference to be found. There is a great difference between post-hoc power and a priori power which estimates the power of the study to detect a predefined effect size of interest using the study sample size. We suggest readers to refer to article by Hoenig and Heisey stating that "because of the one-to-one relationship between p-values and observed power, non-significant p-values always correspond to low powers". ${ }^{4}$ Moreover O’Keefe writes "Second, this [post-hoc] power value provides an answer to question that doesn't much matter, namely: 'What chance was there of producing a statistically significant result, assuming that the population effect is exactly equal to the observed sample effect?",5

As the same information is required to calculate post-hoc power and p-value, the natural outcome is that post-hoc power is inherently associated to the calculated p-value. Post-hoc power of 50\% always provides p-value of 0.05 (Figure). This direct correlation has been reported in statistical literature a number of times. ${ }^{4-6}$ Post-hoc power is nothing more than a report of $\mathrm{p}$-value in a different way and therefore provides no answer to type 2 error. Non-significant studies always have low observed power.

The authors use the search of keys in a house as a common sense example of type 2 error. As the authors state, "reporting the power of a study is analogous to disclosing the extent of a search effort." This is true and in fact is the case in a priori power analysis, ie the probability of finding the keys in prespecified areas of the house. Using study sample size and observed effect size, ie the post hoc power, is only a one-time retrospective look at the data, just like the p-value. 
Instead of reporting the post-hoc power, the scientist could explain how likely it was to observe a significant effect based on the sample size and assumed effect size of interest, similar to reporting the a priori power calculations, as suggested in CONSORT and STROBE guidelines.

Disclosure: The authors declare no conflicts of interest.

Olli Helminen, MD, PhD

Department of Surgery, Central Finland Central Hospital, Jyväskylä, Finland

Cancer and Translational Medicine Research Unit, Medical Research Center Oulu, University of Oulu and Oulu University Hospital, Oulu, Finland

olli.helminen@ksshp.fi

Aleksi Reito, MD, PhD

Department of Surgery, Central Finland Central Hospital, Jyväskylä, Finland

Coxa Hospital for Joint Replacement, Tampere, Finland

aleksi.reito@ksshp.fi

Figure 


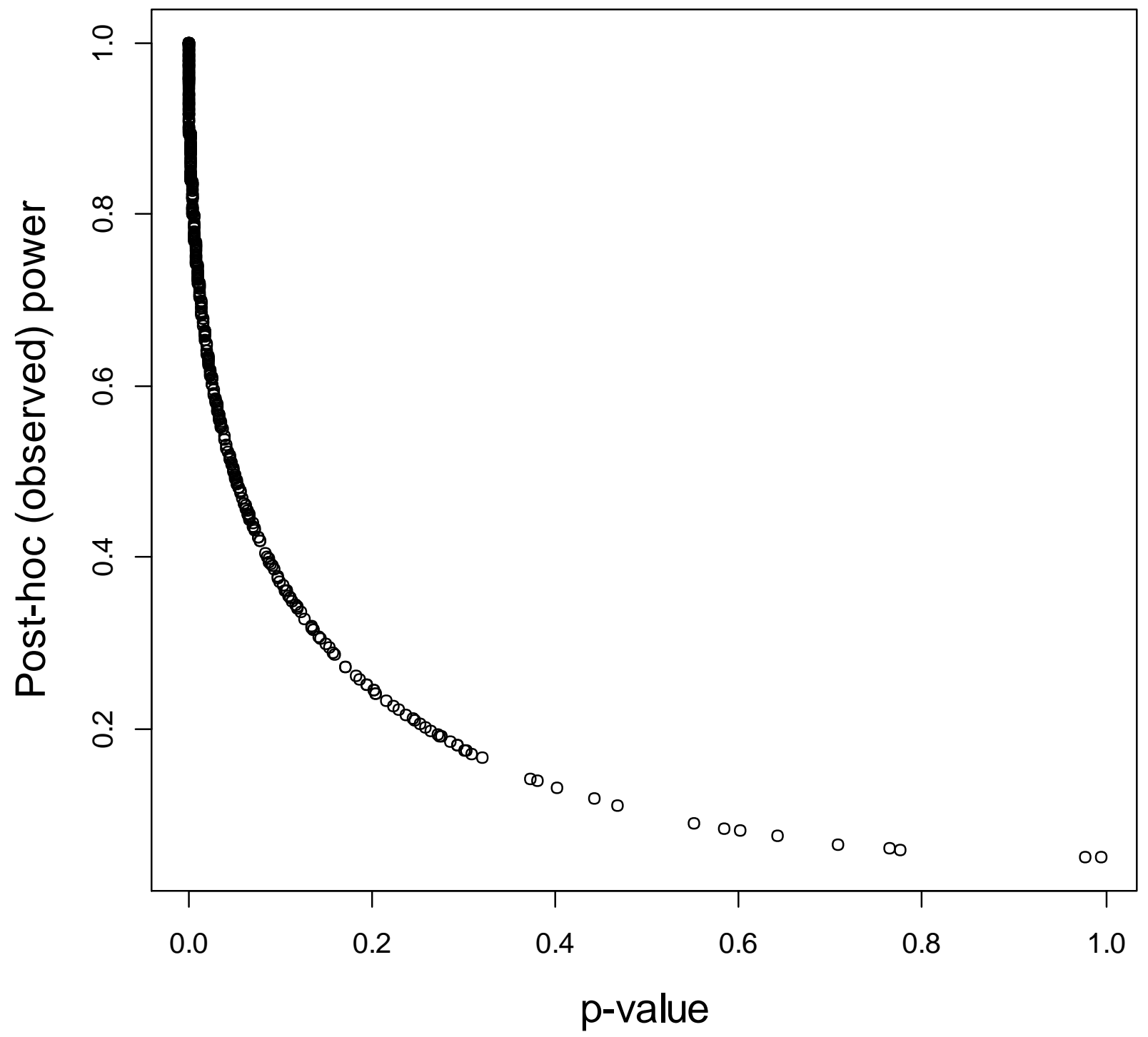

Figure 1: Correlation between observed, ie. post-hoc power and p-value for 500 simulated t-tests with a power of $79.5 \%$ to detect an effect size of 0.5 . p-values higher than 0.05 are more sparse than those $<0.05$.

\section{References}

1. Bababekov YJ, Stapleton SM, Mueller JL, et al. A Proposal to Mitigate the Consequences of Type 2 Error in Surgical Science. Ann Surg 2018;267:621-622. 
2. Button KS, Ioannidis JP, Mokrysz C, et al. Power failure: why small sample size undermines the reliability of neuroscience. Nat Rev Neurosci 2013;14:365-376.

3. Ioannidis JP. Why most published research findings are false. PLoS Med 2005;2:e124.

4. Hoenig J, Heysey D. The Abuse of Power: The Pervasive Fallacy of Power. Calculations for Data Analysis. The American Statistician 2001;55:19-24.

5. O'Keefe DJ. Brief Report: Post Hoc Power, Observed Power, A Priori Power, Retrospective Power, Prospective Power, Achieved Power: Sorting Out Appropriate Uses of Statistical Power Analyses. Communication Methods and Measures 2007;1:291-299.

6. Goodman SN, Berlin JA. The use of predicted confidence intervals when planning experiments and the misuse of power when interpreting results. Ann Intern Med 1994;121:200-206. 